\title{
Pruritus ani and perianal eczema as a manifestation of systemic contact dermatitis
}

\author{
Karolina Hadasik¹, Beata Bergler-Czop ${ }^{1}$, Bartosz Miziołek², Natalia Salwowska², Alina Skrzypek-Salamon ${ }^{2}$
}

${ }^{1}$ Department of Dermatology, School of Medicine, Medical University of Silesia, Katowice, Poland

${ }^{2}$ Department of Dermatology, Andrzej Mielęcki Silesian Independent Public Clinical Hospital, Katowice, Poland

Adv Dermatol Allergol 2017; XXXIV (2): 174-176

DOI:https://doi.org/10.5114/ada.2017.67085

Systemic contact dermatitis (SCD) is a mucocutaneous reaction that results from a systemic distribution of a contact allergen. This condition has been widely reported to occur after the exposure to some drugs (known as baboon syndrome), foods or dental materials. The clinical image is diverse and some cutaneous manifestations may encompass vesicular hand eczema, symmetrical intertriginous and flexural exanthema or lichenoid mucosal eruption in the vulvar area of females. In more severe cases, there is an involvement of large body surfaces that can lead to a development of diffuse scaling dermatitis or even exfoliative erythroderma [1, 2].

The modern lifestyle and progressive industrial development lead to a growing exposure to different metals such as nickel, chromium, cobalt or zinc, what increases the prevalence of contact allergy reactions in the society. Nickel is the most widespread contact allergen in our environment and represents a common component of different alloys, jewelry, foods or kitchen utensils, what undoubtedly makes the way of exposure to this metal very distinguished [3]. It is evident that different food products, such as peanuts, oatmeal, cereals, soy, chocolate, lettuce, broccoli or giblets, contain a relatively high amount of nickel. Interestingly, drinking water also seems to be a significant source of nickel, especially if it is delivered from water tanks of poor quality $[4,5]$.

We present a case of perianal eczema associated with pruritus ani (PA) which occurred in a female patient, with no concomitant systemic disease or any other pathology localized in the rectal area, which resolved following allergen-free diet.

A 42-year-old female patient was admitted to the Dermatology Clinic in Katowice due to persistent anal itching with concomitant eczema in the anogenital area. The patient had suffered from pruritus for over 20 years whereas some skin lesions started to occur in the anogenital area a year before. There was an intense erythema of well-demarcated borders which affected symmetrically both buttocks and the perianal surface. Additionally, some skin maceration and serous exudate could be detected. The previous therapy with both topical steroids and antifungals was ineffective. No concomitant systemic disorders or allergies were previously reported (Figure 1).

A skin biopsy from the perianal area revealed features of a chronic inflammatory process and epidermal proliferation with hyperkeratosis and acantholysis. Laboratory tests towards fungal infections or intestinal parasites were negative. Patch tests (the Polish Baseline Series) revealed a strong hypersensitivity response to nickel (3+). A consultation at the proctologist's detected no malignancies both in digital rectal examination and in rectoscopy. All of common laboratory blood tests were also within the normal ranges.

Although, evident contact with allergens was initially denied by our female patient, a deeper analysis of diet and environmental factors revealed that drinking water was delivered to our patient directly from a drilled well in her garden. Undoubtedly, this source of water had not been previously investigated and presumptively, it could comprise a causative factor for the development of all cutaneous manifestations in our patient. As a consequence, our patient decided to stop using the water from the source in the garden.

A laboratory analysis of water from the drilled well in the garden was ordered by our female patient and a high content of nickel was detected apart from iron and manganese, suggesting the possible source of a contact allergen. In a 3 months' follow-up, cessation of drinking of water from the drilled well and nickel-(allergen)-free diet allowed for a spectacular clinical improvement with a complete resolution of all skin symptoms (Figure 2).

Pruritus ani is a quite common manifestation of several disease entities, including both systemic and derma-

Address for correspondence: Bartosz Miziołek MD, Department of Dermatology, Andrzej Mielęcki Silesian Independent Public Clinical Hospital, 20-24 Francuska St, 40-027 Katowice, Poland, phone: +48 501828 540, e-mail: bmiziolek@gmail.com

Received: 21.01.2016, accepted: 28.04.2016. 
tological conditions. It is a common symptom of different dermatoses involving the perianal area, such as allergic eczema, psoriasis inversa or lichen planus [6]. There are different irritant and allergic factors which can lead to the development of PA and the most common ones are personal care products (soaps, shampoos, recycled toilet paper or intimate wipes) and topical medical agents [7].

A systemic exposure to some allergens has been reported in the English literature to be a significant cause of PA. Giordano-Labadie et al. reported a case of eczema involving perianal and buttocks areas presumptively as a consequence of initial sensitization to previously topically applied sorbic acid contained in the cream. Subsequently, re-exposure to the allergen contained in food resulted in upper skin lesions [8]. Silvestri et al. demonstrated a male patient with a 1.5-year history of treatment-resistant PA that was probably associated with daily peanut butter consumption and a complete resolution of all cutaneous manifestations was obtained with dietary nickel restriction. Interestingly, a specific distribution of skin lesions is speculated to be a consequence of a recall reaction, a phenomenon which involves the reoccurrence of an acute inflammatory skin reaction in previously irradiated skin after the contact with an allergen [9].

In the literature, there are different nickel-free-diet recipes which allow for a resolution of hypersensitivity skin reactions induced by this metal. It is however barely possible to achieve a total dietary elimination of nickel since the substance is widespread and contained in different food products, such as rice, oatmeal, bean, seafood, cacao, nuts, sunflower giblets and canned or frozen

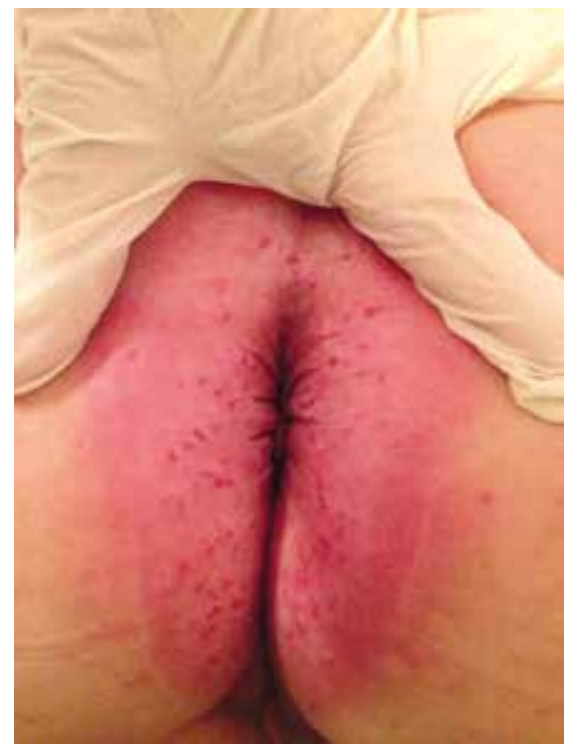

Figure 1. Initial clinical appearance of the perianal skin area food [10]. Interestingly, drinking-water appears also to be a significant source of nickel and its content can be largely varied between water intakes. Essentially, it is obvious that some cooking procedures in metal pots have also an influence on the nickel content in meals, leading to its release in acidic conditions [11].

Interestingly, positive patch tests in patients with PA can be clinically not fully relevant. In a study of Dasan et al., patch tests were performed in 32 patients with PA and positive test results were observed in 18 individuals. The majority of these results were thought not to be clinically relevant and they probably only reflected previous numerous topical treatments [12].

Systemic contact dermatitis involves several different mucocutaneous reactions, which occur in case of exposure to some allergen that could be administered by food intake, parenterally (percutaneously, intravenously) or by inhalation. Both PA and eczema of the perianal area can be manifestations of SCD induced by nickel. Complementary patch tests are recommended in all patients with PA as the next diagnostic step after the exclusion of rectal pathologies, including malignant conditions. The diagnosis of SCD should be considered in all patients with no clinical improvement despite elimination of all known topical sources of nickel. The nickel-free-diet was kept for at least 4 weeks and the resultant clinical improvement confirms the diagnosis of SCD. A diagnosis of SCD is a significant recommendation for continuation of such diet and is management of choice allowing for achievement of clinical remission of the disease.

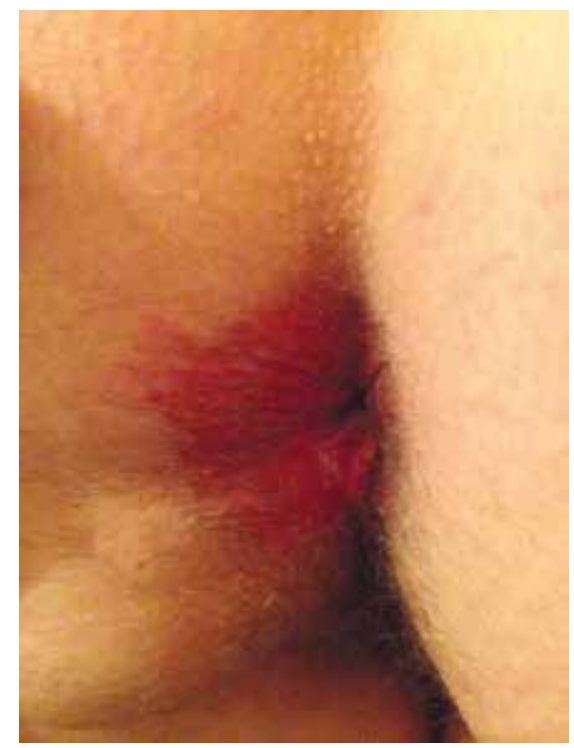

Figure 2. The clinical improvement after 3 months of nickel(allergen)-free diet and cessation of drinking of water from the drilled well in the garden 


\section{Conflict of interest}

The authors declare no conflict of interest.

\section{References}

1. Kulberg A, Schliemann S, Elsner P. Contact dermatitis as a systemic disease. Clin Dermatol 2014; 32: 414-9.

2. Fabbro SK, Zirwas MJ. Systemic contact dermatitis to foods: nickel, BOP, and more. Curr Allergy Asthma Rep 2014; 14: 463.

3. Basko-Plluska JL, Thyssen JP, Schalock PC. Cutaneous and systemic hypersensitivity reactions to metallic implants. Dermatitis 2011; 22: 65-79.

4. Darsow U, Fedorov M, Schwegler U, et al. Influence of dietary factors, age and nickel contact dermatitis on nickel excretion. Contact Dermatitis 2012; 67: 351-8.

5. Roßkamp E, Kölle W. Vorkommen, Bedeutung und Nachweis von Nickel. In: Die Trinkwasserverordnung. Grohmann A, Hässelbarth U, Schwerdtfeger W (eds). Schmidt Erich Verlag, Berlin 2003; 329-35.

6. Nasseri YY, Osborne MC. Pruritus ani: diagnosis and treatment. Gastroenterol Clin North Am 2013; 42: 801-13.

7. Smith LE, Henrichs D, McCullah RD. Prospective studies on the etiology and treatment of pruritus ani. Dis. Colon Rectum 1982; 25: 358-63.

8. Giordano-Labadie F, Pech-Ormieres C, Bazex J. Systemic contact dermatitis from sorbic acid. Contact Dermatitis 1996; 34: 61-2.

9. Silvestri DL, Barmettler S. Pruritus ani as a manifestation of systemic contact dermatitis: resolution with dietary nickel restriction. Dermatitis 2011; 22: 50-5.

10. Petrucci F, Bocca B, Forte $G$, et al. Role of diet in nickel dermatitis. The Open Chem. Biomed Methods 2009; 2: 55-7.

11. Sharma AD. Low nickel diet in dermatology. Indian J Dermatol 2013; 58: 240.

12. Dasan S, Neill SM, Donaldson DR, Scott HJ. Treatment of persistent pruritus ani in a combined colorectal and dermatological clinic. Br J Surg 1999; 86: 1337-40. 